\title{
Diagnostic Laparoscopy in the Evaluation of Female Factors in Infertility in Kashmir Valley
}

\author{
Qurat-ul-Ain Wani ${ }^{1}$, Rifat Ara ${ }^{2}$, Sajad Ahmad Dangroo ${ }^{3 *}$, Mehbooba Beig 4
}

\section{Article History:}

Received 10 October 2013

Accepted 7 January 2014

Revised 25 December 2013

Available online 7 January 2014

\section{Keywords:}

Diagnostic Laparoscopy

Infertility

Kashmir

Primary Infertility

Secondary Infertility

\section{Corresponding Author:}

Sajad Ahmad Dangroo, Senior Resident department of General and Minimal Access Surgery, Sheri Kashmir Institute of Medical Sciences Srinagar, J\&K, India. Tel: +919906665623

Email: drsajadskims@gmail.com

\begin{abstract}
Objectives: To evaluate the female factors in infertility using laparoscopy in Kashmiri women.

Materials and Methods: One hundred cases of infertility [primary and secondary] were included in this prospective study. Before the procedure, apart from complete history and detailed examination, baseline investigations (complete blood count, blood sugar, kidney and liver function tests, ECG, chest $\mathrm{X}$-ray) were performed as per our institutional protocol for pre-anaesthesia check up. Laparoscopy was done in proliferative phase of menstrual cycle. To test the patency of tubes, chromotubation was done in all cases under laparoscopic vision by using 10-15 ml of autoclaved methylene blue dye. All the data was collected on pre-designed proforma and the results were tabulated and raw percentages calculated to describe the results.

Results: In primary infertility group [n=82], most common laparoscopic finding was tubal occlusion in 15 (18.3\%), followed by endometriotic deposits in 14 (17.1\%) patients. Other findings were polycystic ovaries in 10 patients $(12.2 \%)$, peritubal and periovarian adhesions in 6 patients (7.3\%), fibroids in 6 patients (7.3\%), genital tract tuberculosis in 5 patients $(6.1 \%)$, ovarian cysts in 4 patients $(4.8 \%)$, hypoplastic uterus in 2 patients (2.4\%) and pelvic inflammatory diseases in 2 patients $(2.4 \%)$, in this group. The commonest finding by laparoscopy in patients with secondary infertility $[n=18]$ was tubal occlusion in $5(27.7 \%)$, followed by peritubal and periovarian adhesions in $4(22.2 \%)$ patients.

Conclusion: laparoscopy is very effective and cheap method in evaluating infertile women and should be considered earlier in infertility workup for effective and early treatment decisions.
\end{abstract}

1- Senior Resident department of obstetrics and gynecology, Sheri Kashmir Institute of Medical Sciences Medical College, Srinagar, J\&K, India.

2- Associate Professor department of obstetrics and gynecology, Sheri Kashmir Institute of Medical Sciences Medical College, Srinagar, J\&K,India.

3- Senior Resident department Of General and Minimal Access Surgery, Sheri Kashmir Institute of Medical Sciences Srinagar, J\&K, India.

4- Assistant Professor department of obstetrics and gynaecology, Sheri Kashmir Institute of Medical Sciences Medical College, Srinagar, J\&K, India. 


\section{Introduction:}

Infertility is best defined as the inability to conceive after one year of unprotected regular intercourse $(1,2)$. Based on this, $60-$ 80 million couples all over the world can be labelled as suffering from infertility (3). Estimates of infertility vary widely among Indian states from 3.7 per cent in Uttar Pradesh, Himachal Pradesh and Maharashtra (4), to 5 per cent in Andhra Pradesh (5) and 15 per cent in Kashmir (6). Infertility affects men and woman alike, as both genders report associated psychological distress, depression and low self-esteem $(7,8)$. In many cultures, the social repercussions of infertility compound the individual impact. Infertility has been observed to result in divorce, loss of economic resources, and even the annulment of rights to burial grounds (9).

The common factors responsible for infertility in females are anovulatory disorder, tubal factors, endometriosis, uterine and cervical factors (10). Untreated pelvic inflammatory diseases (PID), postabortal, postpartum infections and tuberculosis are common factors of infertility in developing countries (11). Conventional way to assess the uterine cavity, tubal structure and tubal patency was Hysterosalpingography but it has now been largely superseded by laparoscopy and hysteroscopy. Advances in endoscopic surgery have revolutionized the approaches of obstetricians for diagnosis and management of patients with infertility. Laparoscopy is the standard means of diagnosing the tubal pathology, peritoneal factors and endometriosis and intraabdominal causes of infertility. Not only does this help in identification of unsuspected pelvic pathology but also contributes to decision making of infertility treatment.

In Kashmir most of the patients are illiterate and from low socioeconomic status. They go to untrained health practitioners for the infertility treatment, which leads to delay in proper management. The role of laparoscopy in the diagnosis of primary and secondary infertility is established beyond any doubt. An effort was made to evaluate the female factors in infertility in Kashmiri women using laparoscopy and to detect the diagnostic efficacy of laparoscopy.

\section{Material \& Methods:}

This prospective study was conducted in the department of Obstetrics and Gynaecology, Sher-i-Kashmir Institute of Medical Sciences Medical College Hospital, Srinagar, Jammu and Kashmir, India from December 2011 to April 2013 after approval from the human ethical committee of our institute. One hundred eleven (111) infertile patients attended the gynaecology department of our institute from December 2011 to April 2013. One hundred cases of infertility (primary and secondary) were selected for diagnostic laparoscopy. Those patients who had absolute or relative contraindications for anaesthesia or laparoscopy were excluded from the study. Proper informed written consent was taken from every patient. All the patients were admitted in the ward one day prior to procedure. Before the procedure, apart from complete history and detailed examination, baseline investigations (complete blood count, blood sugar, kidney and liver function tests, ECG, chest X-ray) were performed as per our institutional protocol for pre-anaesthesia check up. Laparoscopy was done in proliferative phase of menstrual cycle. To test the patency of tubes, chromotubation was done in all cases under laparoscopic vision by using 10-15 ml of autoclaved methylene blue dye. All the data was collected on pre-designed proforma and the results were tabulated and raw percentages calculated to describe the results.

\section{Results:}

We studied one hundred cases of female infertility comprising of 82 (82\%) cases of primary and $18(18 \%)$ cases of secondary infertility. $38(46.3 \%)$ cases of primary infertility were in the age group of 20-30 years followed by $29(35.4 \%)$ cases in the age group of $31-40$ years. In secondary infertility group $11(61.1 \%)$ cases were in the age group of 31-40 years and 7 (38.8\%) cases in 20-30 years age group. The mean age at presentation in our study was 25 years in primary and 31 years in secondary infertility group (Table 1). 
The duration of infertility ranged from 1-15 years. The mean duration of infertility was 4.6 years in primary and 5.2 years in secondary infertility group. Maximum number of cases had duration of infertility between 1-5 years, in both primary infertility group- 41 cases (50\%) and secondary infertility group- 10 cases (55.5\%) (Table 2).

Fourteen patients (17.1\%) of primary infertility group were asymptomatic. The main associated symptoms in this group were dysmenorrhea in 32 (39.02\%), dyspareunia in 13 (15.8\%), chronic pelvic pain in $9(10.9 \%)$, menorrhagia in $5(6.09 \%)$ and hirsutism in $2(2.4 \%)$ patients. Among the 18 patients with secondary infertility, 4 patients $(22.2 \%)$ were asymptomatic. In this group, the main associated symptom was dyspareunia in $6(33.3 \%)$ patients. Other symptoms like irregular cycles were seen in 2 patients $(11.1 \%)$, dysmenorrhea in 2 patients (11.1\%), menorrhagia in 2 patients (11.1\%), chronic pelvic pain in 1 patient (5.5\%) and hirsutism in 1 patient (5.5\%) in this group (Table 3 ).

Laparoscopy did not demonstrate evidence of any pathology in 20 (20\%) patients out of 100 patients in our study. In primary infertility group, most common laparoscopic finding was tubal occlusion in 15 (18.3\%), followed by endometriotic deposits in 14 (17.1\%) patients. Other findings were polycystic ovaries in 10 patients (12.2\%), peritubal and periovarian adhesions in 6 patients $(7.3 \%)$, fibroids in 6 patients (7.3\%), genital tract tuberculosis in 5 patients $(6.1 \%)$, ovarian cysts in 4 patients (4.8\%), hypoplastic uterus in 2 patients $(2.4 \%)$ and pelvic inflammatory diseases in 2 patients (2.4\%), in this group. The commonest finding by laparoscopy in patients with secondary infertility was tubal occlusion in 5 (27.7\%), followed by peritubal and periovarian adhesions in 4 (22.2\%) patients (Table 4).

There were no complications in 37 (45.1\%) and $6(33.3 \%)$ patients of primary and secondary infertility respectively. The complications after laparoscopy in our study are shown in Table 5. All the complications were of mild grade and did not require any active intervention.

\section{Discussion:}

The World Health Organization (WHO) estimates that 60 to 80 million couples worldwide currently suffer from infertility (12). Infertility varies across regions of the world and is estimated to affect 8 to 12 per cent of couples worldwide $(13,14)$. The WHO estimates the overall prevalence of primary infertility in India to be between 3.9 and 16.8 per cent (12). The female factors contribute most (i.e. $40-55 \%$ ) in the etiologies of infertility followed by male factors (30-40\%), both partners (10\%) and unexplained (10\%) (15). Infertility rates in Kashmir are very high as compared to other states of India (6).

The mean age at presentation in our study was 25 years in primary and 31 years in secondary infertility. Many studies in the literature have shown that there is rise in age at which women presented with infertility. In our study, 29 (35.4\%) patients presenting with primary and 11 (61.1\%) patients with secondary infertility were of age $>35$ years. Because of the decline in fertility and the increased time to conception that occurs after the age of 35 , women $>35$ years of age should be referred for infertility work-up after 6 months of trying to conceive (16). The duration of infertility was 1-5 years in the majority of patients $(50 \%)$ in primary and (55.5\%) in secondary infertility. The mean duration of infertility was found to be 4.6 years in primary and 5.2 years in secondary infertility. Boricha et al observed similar results (17).

Major symptoms in our study were dysmenorrhoea, dyspareunia and irregular cycles, which are in accordance with other infertility studies (18). These symptoms were found to be frequently associated with organic pelvic pathology. The diagnostic laparoscopy should be considered early in symptomatic patients during infertility workup (19).

Tubal disease accounts for $15-20 \%$ of cases of primary infertility and approximately $40 \%$ of secondary infertility (20). Pelvicperitoneal adhesions (mostly sequels of prior infections from organisms like Chlamydia trachomatis and Neisseria gonorrhea) constitute the single most common class of tubal pathology 
responsible for tubal infertility (21). They cause anatomic and physiological dysfunction of tubes and prevent ovum pickup, fertilization and zygote transport between the ovary and the uterus in the normal process of procreation $(22,23)$. In our study, tubal blockage was present in $18.3 \%$ in the primary and $27.7 \%$ in the secondary infertility group and peritubal and periovarian adhesions in $7.3 \%$ and $22.2 \%$ of primary and secondary infertility respectively.

In the United States, more than 1 million women are treated for PID every year, and 200,000 to 300,000 of them require hospitalization. The situation is even worse in developing countries where health care is not readily accessible. A single episode of PID carries up to $10 \%$ risk of future tubal factor infertility. In our study, PID was present in $2.4 \%$ and $11.1 \%$ cases of primary and secondary infertility respectively. In the study conducted by Raida M and co-workers, PID was found in $2.13 \%$ of primary and $5.08 \%$ of secondary infertility patients (24). In our study, pelvic inflammatory diseases (PID) and tubal blockage were more frequently found in secondary infertility as compared to primary infertility. Talat $\mathrm{N}$ et al (25) have reported same results.

Polycystic ovarian disease causes hormonal imbalance in women that is thought to be one of the leading causes of female infertility. Polycystic ovarian syndrome causes more than $75 \%$ of cases of anovulatory infertility (26).The prevalence of polycystic ovarian disease (PCOD) in asymptomatic women is thought to be between 16 and 33\% (27). The incidence of polycystic ovaries in our study was $12.1 \%$ in case of primary infertility and no case of it was found in secondary infertility group.

The incidence of myoma in women with infertility without any obvious cause of infertility is estimated to be $1-2.4 \%$ (28). In our study, fibroids were seen in $7.3 \%$ and $5.5 \%$ cases of primary and secondary infertility respectively that correlated with study conducted by Aziz N et al (29).

Although exact prevalence of endometriosis in general population of reproductive age is not known, it is believed to be in the range of $3-10 \%$ (30). In our study, endometriosis was seen in $17.1 \%$ and $11.1 \%$ cases of primary and secondary infertility respectively. Muzii $\mathrm{L}$ et al in their study demonstrated that endometriosis occurs three times more often in patients with primary infertility than in patients with secondary infertility (31).

Genital tuberculosis is an important cause of infertility especially in endemic zones. Genital tuberculosis not only causes tubal obstruction and dysfunction but also impairs implantation due to endometrial involvement and ovulatory failure from ovarian involvement (32). The prevalence of female genital tract tuberculosis in infertility clinics shows marked variations in different countries ranging between 15 and 25\% (33). Our study revealed genital tract tuberculosis in $6.1 \%$ and $5.5 \%$ cases of primary and secondary infertility respectively. In our study, hypoplastic uterus was seen in 2 $(2.4 \%)$ patients of primary infertility.

Laparoscopy was not able to demonstrate any pathology in $20(20 \%)$ cases out of 100 cases in our study. Out of these, 4 patients were diagnosed as having male factor infertility on subsequent follow up. 16 $(16 \%)$ patients had unexplained infertility with no obvious cause seen. Present study correlated with observations of the other studies (17) suggesting that laparoscopy was useful in achieving correct diagnosis in majority of the patients. In our study, laparoscopy was able to correctly diagnose pathology in $62(75.6 \%)$ patients.

In 2007, Nakagawa $K$ et al strongly recommended diagnostic laparoscopy for unexplained infertile patients because of the high rate of abnormal findings (87\%) on laparoscopy (34). In a cross-sectional study in 2009, Kahyaoglu $S$ et al evaluated the treatment strategy change after diagnostic laparoscopy for primary and secondary unexplained infertile patients. The rate of pelvic abnormalities related to infertility was found to be $60 \%$ and $69 \%$ among primary and secondary infertile patients that was reflected with a rate of $43 \%$ and $49 \%$ of treatment strategy change following laparoscopy respectively (35).

The incidence of postoperative complications with laparoscopy in our study was very low which corresponds with the 
findings of few other national and international studies $(36,37,38)$. There were no complications in $37(45.1 \%)$ and 6 (33.3\%) patients of primary and secondary infertility respectively. All the complications were of mild grade and did not require any active intervention.

Diagnostic laparoscopy by direct visualization of pelvic structures facilitates identification of etiology which commonly includes endometriosis, adhesions, ovarian cysts or pelvic inflammatory diseases, so that a therapeutic intervention can be initiated, while avoiding potentially ineffective or unnecessary empiric medical treatment for ovulation induction. In some patients, diagnostic laparoscopy alters treatment plans, including earlier utilization of assisted reproductive technology. Thus, diagnostic laparoscopy can be safely used earlier in the evaluation of infertile females.

\section{Conclusion :}

Keeping in view the high infertility rates and illiteracy in our region, proper guidance and education of infertile females is needed to consult earlier at proper infertility clinics. Laparoscopy is very effective method in evaluating these infertile women to prevent delay in management. Diagnostic laparoscopy should be considered earlier in women with history of pelvic inflammatory diseases, pelvic surgery and chronic pelvic pain for effective treatment decisions. It may be considered in appropriately selected infertile patients even after normal findings, as important pelvic pathology may be identified in a significant number of patients. It is most useful in diagnosing cases with endometriosis and tubal factor infertility.

\section{Conflicts of interest:}

We declare that no conflicts of interests exist regarding the material in this manuscript.

\section{Acknowledgments:}

We have no acknowledgements to disclose. 
Table 1. Various age groups analyzed in the study and their respective percentages of infertility occurrence.

\begin{tabular}{ccccc}
\hline $\begin{array}{c}\text { Age group } \\
\text { (yrs) }\end{array}$ & \multicolumn{2}{c}{$\begin{array}{c}\text { Primary } \\
\text { infertility }(\mathrm{n}=82)\end{array}$} & \multicolumn{2}{c}{$\begin{array}{c}\text { Secondary } \\
\text { infertility }(\mathrm{n}=18)\end{array}$} \\
\hline & $($ no) & $(\%)$ & $($ no $)$ & $(\%)$ \\
\cline { 2 - 5 }$<20$ & 15 & 18.3 & 0 & 0 \\
$20-30$ & 38 & 46.3 & 7 & 38.8 \\
$31-40$ & 29 & 35.4 & 11 & 61.1 \\
\hline
\end{tabular}

Table 2. Distribution of cases according to duration of infertility at the time of presentation.

\begin{tabular}{ccccc}
\hline Duration of infertility (yrs) & \multicolumn{2}{c}{ Primary infertility $(\mathrm{n}=82)$} & \multicolumn{2}{c}{ Secondary infertility $(\mathrm{n}=18)$} \\
\cline { 2 - 5 } & $(\mathrm{no})$ & $(\%)$ & $(\mathrm{no})$ & $(\%)$ \\
\cline { 2 - 5 } $1-5$ & 41 & 50 & 10 & 55.5 \\
$6-10$ & 35 & 42.7 & 5 & 27.7 \\
$11-15$ & 6 & 7.3 & 3 & 16.6 \\
\hline
\end{tabular}

Table 3. Presenting symptoms of patients analyzed in the study and their respective percentages in primary and secondary infertility groups.

\begin{tabular}{lcccc}
\hline \multicolumn{1}{c}{ Symptoms } & Primary infertility & $(\mathrm{n}=82)$ & Secondary infertility $(\mathrm{n}=18)$ \\
\cline { 2 - 5 } & $(\mathrm{no})$ & $(\%)$ & $(\mathrm{no})$ & $(\%)$ \\
Irregular cycles & 7 & 8.5 & 2 & 11.1 \\
Dysmenorrhea & 32 & 39.02 & 2 & 11.1 \\
Dyspareunia & 13 & 15.8 & 6 & 33.3 \\
Chronic pelvic pain & 9 & 10.9 & 1 & 5.5 \\
Menorrhagia & 5 & 6.09 & 2 & 11.1 \\
Hirsutism & 2 & 2.4 & 1 & 5.5 \\
Asymptomatic & 14 & 17.1 & 4 & 22.2 \\
\hline
\end{tabular}


Table 4. Findings on diagnostic laparoscopy in the study and their respective percentages in primary and secondary infertility groups.

\begin{tabular}{|c|c|c|c|c|}
\hline \multirow[t]{2}{*}{$\begin{array}{l}\text { Findings on Diagnostic } \\
\text { laparoscopy }\end{array}$} & \multicolumn{2}{|c|}{$\begin{array}{c}\text { Primary infertility } \\
(\mathrm{n}=82)\end{array}$} & \multicolumn{2}{|c|}{ Secondary infertility $(n=18)$} \\
\hline & (no) & $(\%)$ & (no) & $(\%)$ \\
\hline $\begin{array}{l}\text { No pathology } \\
\text { demonstrated }\end{array}$ & 18 & 21.9 & 2 & 11.1 \\
\hline Tubal occlusion & 15 & 18.3 & 5 & 27.7 \\
\hline $\begin{array}{l}\text { Peritubal/Periovarian } \\
\text { adhesions }\end{array}$ & 6 & 7.3 & 4 & 22.2 \\
\hline Ovarian cysts & 4 & 4.9 & 1 & 5.5 \\
\hline Endometriotic deposits & 14 & 17.1 & 2 & 11.1 \\
\hline $\begin{array}{l}\text { Genital tract } \\
\text { tuberculosis }\end{array}$ & 5 & 6.1 & 1 & 5.5 \\
\hline Hypoplastic uterus & 2 & 2.4 & 0 & 0 \\
\hline Fibroids & 6 & 7.3 & 1 & 5.5 \\
\hline Polycystic ovaries & 10 & 12.2 & 0 & 0 \\
\hline $\begin{array}{l}\text { Pelvic inflammatory } \\
\text { diseases }\end{array}$ & 2 & 2.4 & 2 & 11.1 \\
\hline
\end{tabular}

Table 5. Complications after diagnostic laparoscopy in the study and their respective percentages in primary and secondary infertility groups.

\begin{tabular}{lcccc} 
Complications after laparoscopy & \multicolumn{2}{c}{ Primary infertility $(\mathrm{n}=82)$} & \multicolumn{2}{c}{ Secondary infertility $(\mathrm{n}=18)$} \\
\cline { 2 - 5 } & $(\mathrm{no})$ & $(\%)$ & $(\mathrm{no})$ & $(\%)$ \\
Nausea/vomiting & & & & \\
Fever & 19 & 23.17 & 5 & 27.7 \\
Surgical emphysema & 11 & 13.41 & 3 & 16.6 \\
Shoulder tip pain & 2 & 2.4 & 1 & 5.5 \\
Abdominal pain & 9 & 10.9 & 2 & 11.1 \\
No complications & 4 & 4.8 & 1 & 5.5 \\
\hline
\end{tabular}




\section{References:}

1. Hammond MG. Evaluation of the infertile couple. Obstet Gynecol Clin North Am. 1987; $14: 821-30$.

2. Taylor A. ABC of sub fertility: Making a diagnosis. BMJ. 2003;327:494-7.

3. Fathalla MF. Reproductive health: A global overview. Early Human Develop. 1992;29:3542.

4. Talwar PP, Go OP, Murali IN. Prevalence of infertility in different population groups in India and its determinants. In: Statistics and demography. New Delhi: National Institute

of Health \& Family Welfare \& Indian Council of Medical Research; 1986.

5. Unisa S. Childlessness in Andhra Pradesh, India: Treatment-seeking and consequences. Reprod Health Matters. 1999; 7:54-64.

6. Zargar AH, Wani AI, Masoodi SR, Laway BA, Salahuddin M. Epidemiologic and etiologic aspects of primary infertility in the Kashmir region of India. Fertil Steril. 1997;68:637-43.

7. Chachamovich JR, Chachamovich E, Ezer H, Fleck MP, Knauth D, Passos EP. Investigating quality of life and health-related quality of life in infertility: a systematic review. J Psychosom Obstet Gynaecol. 2010;31(2):101-110.

8. Cui W: Mother or nothing: the agony of infertility. Bull World Health Organ. 2010;88:881882.

9. Greil AL, Slauson-Blevins K, McQuillan J: The experience of infertility: a review of recent literature. Sociol Health Illn. 2010;32:140 162.

10. Jose Miller AB, Boyden JW, Frey KA. Infertility. Am Fam Physician. 2007;75:849-56.

11. Rehana R, Majid SS. Aetiological factors of infertility. J Postgrad Med Inst. 2004; 18:16671.

12. Shea O. Rutstein, ORC Macro, Iqbal H. Shah. Infecundity, infertility, and childlessness in developing countries. DHS Comparative Reports No 9. Calverton, Maryland, USA: ORC Macro and the World Health Organization; 2004.

13. Sciarra J. Infertility: an international health problem. Int J Gynaecol Obstet. 1994; 46:15563.

14. Looking back, looking forward: a profile of sexual and reproductive health in India. New Delhi: Population Council. Population Council. Infertility. 2004; 67-72.

15. Speroff L, Marc A. F. Female infertility: Clinical Gynecologic Endocrinology and Infertility, 7th Ed, Jaypee Brothers Medical Publishers, India. 2005; 1013-1068. 
16. Liu K, Case A. Advanced reproductive age and fertility. J Obstet Gynaecol Can. 2011; 33(11):1165-75

17. Boricha Y.G, Sharma R.K, Boricha B.G, Sabrina Mhapankar, Archana Chaterjee, Narshetty J. laparoscopy in 50 infertile couples: Prospective study. International Journal of Medical and Clinical Research. 2011;2(2): 63-66.

18. Aziz N. Laparoscopic evaluation of female factors in infertility. J Coll Physicians Surg Pak. 2010;20(10):649-52.

19. Milingos S, Protopapas A, Kallipolitis G, Drakakis P, Makrigiannakis A, Liapi A, et al. Laparoscopic evaluation of infertile patients with chronic pelvic pain. Reprod Biomed Online. 2006;12(3):347-53.

20. Edmonds K, editor. Dewhurst's textbook of obstetrics and gynaecology. 7th ed. India: Blackwell publishing; 2007.

21. Otolorin EO, Ojengbede O, Falase AO. Laparoscopic evaluation of the tuboperitoneal factor in infertile Nigerian women. Int J Gynaecol Obstet. 1987; 25(1):47-52.

22. Marana R, Catalano GF, Muzii L, Caruana P, Margutti F, Mancuso S. The prognostic role of salpingoscopy in laparoscopic tubal surgery. Hum Reprod. 1999;14(12):2991-5

23. Honore GM, Holden AE, Schenken RS. Pathophysiology and management of proximal tubal blockage. Fertil Steril. 1999; 71(5):785-95.

24. Al-Wazzan R.M, Jabbar E. Diagnostic laparoscopy in female infertility. Ann. Coll. Med. Mosul. 2009; 35(1):58-64.

25. Talat N, Lubna H, Gulmeen, Farrah N, Shahida S. Laparoscopic evaluation in infertility. J Coll Physicians Surg Pak. 2009;19:704-7.

26. Gorry A, White DM, Franks S. Infertility in polycystic ovary syndrome: focus on lowdose gonadotropin treatment. Endocrine.2006; 30(1):27-33.

27. Enda McVeigh. Polycystic ovarian syndrome. In: Baker PN, Leusley DM, editors. Obstetric and gynaecology: an evidence based text for Mrocg. London: Oxford University Press. 2004; 588-93.

28. Khaula K. Role of diagnostic laparoscopy in evaluation of female sub fertility. Serv Inst Med Sci. 2005;3:31-4.

29. Aziz N. Laparoscopic evaluation of female factors in infertility. J Coll Physicians Surg Pak. 2010;20(10):649-52.

30. Itdsa JS, Rock JA. Endometriosis in Linde TE (Editor) Operative gynaecology Lippincott Williams Wilkins. 2003; 9:595-38. 
31. Muzii L, Marana R, Brunetti L, Orlando G, Michelotto B, Benedetti Panici P. Atypical endometriosis revisited clinical and biochemical evaluation of different forms of superficial implants. Fertil Steril. 2000; 74(4):739-742

32. Roy H, Roy S, Roy S. Use of polymerase chain reaction for diagnosis of endometrial tuberculosis in high risk sub fertile women in an endemic zone. J Obstet Gynecol India. 2003;53:260-3.

33. Varma TR. Genital tuberculosis and subsequent fertility. Int J Gynaecol Obstet. 1991;35(1):1-11.

34. Nakagawa K, Ohgi S, Horikawa T, Kojima R, Ito M, Saito H. Laparoscopy should be strongly considered for women with unexplained infertility. J Obstet Gynaecol Res. 2007;33(5):665-70.

35. Kahyaoglu S, Kahyaoglu I, Yilmaz B, Var T, Ertas IE, Mollamahmutoglu L, et al. Should diagnostic laparoscopy be performed initially or not, during infertility management of primary and secondary infertile women? A cross-sectional study. J Obstet Gynaecol Res. 2009;35(1):139-44.

36. Marcoux S, Maheux R, Bérubé S. Laparoscopic surgery in infertile women with minimal or mild endometriosis. N Eng J Med. 1997;337(4):217-22.

37. Cahill DJ, Wardle PG. Management of infertility. BMJ. 2002; 325:28-32.

38. Popovic J, Sulovic V, Vucetic D. Laparoscopy treatment of adnexal sterility. Clin Exp Obstet Gynecol. 2005;32(1):31-4. 